\title{
Spin-orbit interaction in curved graphene ribbons
}

\author{
D. Gosálbez-Martínez, ${ }^{1}$ J. J. Palacios, ${ }^{1,2}$ and J. Fernández-Rossier ${ }^{1}$ \\ ${ }^{1}$ Departamento de Física Aplicada, Universidad de Alicante, San Vicente del Raspeig, Spain \\ ${ }^{2}$ Departamento de Física de la Materia Condensada, Universidad Autónoma de Madrid, Spain \\ (Received 1 December 2010; revised manuscript received 14 January 2011; published 16 March 2011)
}

\begin{abstract}
We study the electronic properties of electrons in flat and curved zigzag graphene nanoribbons using a tight-binding model within the Slater Koster approximation, including spin-orbit interaction. We find that a constant curvature across the ribbon dramatically enhances the action of the spin-orbit term, strongly influencing the spin orientation of the edge states: Whereas spins are normal to the surface in the case of flat ribbons, this is no longer the case for curved ribbons. This effect is very pronounced, the spins deviating from the normal to the ribbon, even for very small curvature and a realistic spin orbit coupling of carbon. We find that curvature results also in an effective second neighbor hopping that modifies the electronic properties of zigzag graphene ribbons. We discuss the implications of our findings in the spin Hall phase of curved graphene ribbons.
\end{abstract}

DOI: 10.1103/PhysRevB.83.115436

PACS number(s): 81.05.ue, 71.70.Ej

\section{INTRODUCTION}

The electronic structure of graphene depends both on its structure at the atomic scale, determined by the $s p^{2}$ hybridization, and on its structure at a much larger length scale, determined the shape of the sample. ${ }^{1,2}$ Thus, the electronic properties of flat graphene differ in subtle but important ways from rippled graphene ${ }^{3}$ and the properties of carbon nanotubes are fully determined by the way they fold. ${ }^{1}$ Curvature is believed to affect the transport, ${ }^{4,5}$ magnetic, ${ }^{6}$ and spin relaxation properties of graphene. ${ }^{7}$ In a wider context, the interplay between mechanical deformations and electronic properties, the so-called flexoelectronics, is giving rise to a new branch in nanotechnology. Whereas conventional electronics devices are based on the capability to tune their working properties by application of external perturbations in the form of electric and magnetic fields, mechanical deformation can have a major impact on the properties of nanoelectronic devices. This results in a wide range of new effects, like piezoelectric nanogenerators ${ }^{8}$ and field effects induced by piezoelectric effects ${ }^{9}$ in $\mathrm{ZnO}$ nanowires, and stress driven Mott transitions in $\mathrm{VO}_{2}$ nanowires. ${ }^{10}$

The spintronic and magnetic properties of graphene are intriguing. From the theory side, there are two bold predictions. First, graphene should display a quantum spin Hall phase with spin-filtered states in the zigzag edges. ${ }^{11,12}$ Second, the same edges should have ferromagnetic order. ${ }^{13-22}$ The striking progress in the fabrication of atomically precise graphene ribbons is starting to provide experimental evidence for the existence of stable zigzag edges. ${ }^{23-25}$ On the other hand, several groups have reported the observation of ferromagnetic order in graphene and graphite, ${ }^{26-30}$ in most instances in samples that contain many flakes or structural disorder, or have been irradiated.

The observation of the spin Hall phase in flat graphene would require reducing the temperature below the spin-orbit induced gap, which is smaller than $10 \mu \mathrm{eV} \cdot{ }^{31-33}$ From this point of view it would be desirable to increase the strength of spin-orbit interaction in graphene. Hints of how this could be achieved come from experiments. On one side, the spin relaxation time of graphene, as measured in lateral spin valves, is in the range of $100 \mathrm{ps},{ }^{34}$ much shorter than expected from the small size of spin-orbit and hyperfine nuclear coupling. ${ }^{35}$ Thus, some mechanism enhancing the strength of spin-orbit interaction must be at play in these samples. A possible candidate could be curvature ${ }^{7,36}$ induced by ripples and adatoms. ${ }^{37,38}$ Curvature has been shown to enhance the effect of spin-orbit coupling in the case of carbon nanotubes, for which recent experimental work has reported zero field splittings induced by spin-orbit coupling splittings in the range of $200 \mu \mathrm{eV} .^{36}$

The recently reported fabrication of curved graphene ribbons by unzipping carbon nanotubes ${ }^{39-41}$ opens the way toward the experimental study of the effect of curvature on the edge states of graphene ribbons. Here we study this system from the theoretical point of view and we compare the spin properties of a graphene ribbon both for flat and curved ribbons. In flat graphene the $\pi$ bands are decoupled from the $\sigma$ bands, unless spin-orbit coupling is considered. However, the effect of spin-orbit coupling on $\pi$ bands occurs only via virtual transition to higher energy $\sigma$ bands.

In the case of flat graphene, it has been verified that the effect of spin-orbit on the $\pi$ bands can be properly described by an effective spin dependent second neighbor hopping between the $\pi$ orbitals. This is the so called Kane and Mele model, ${ }^{11,12}$ which predicts that graphene is a quantum spin Hall insulator with a spin and valley dependent gap and peculiar spin-filter zigzag edge states. ${ }^{11,12}$ In the case of curved graphene, $\pi$ and $\sigma$ orbitals are coupled, and to the best of our knowledge the validity of the Kane-Mele model has not been tested. This is why we adopt a different strategy ${ }^{7,31,42-45}$ and use a four-orbital tight-binding model, which includes both the $\pi$ orbitals and the $s, p_{x}$, and $p_{y}$ orbitals.

The rest of this paper is organized as follows. In Sec. II we describe the tight-binding method used in our calculations and review some general results about the spin properties of the system. In Sec. III we present results for the electronic structure of flat zigzag graphene ribbons and compare with those of the Kane-Mele model. In Sec. IV we address the main point of this work, the electronic structure of edge states in curved graphene zigzag ribbons. In Sec. V we discuss our results. 


\section{FORMALISM}

In this section we briefly comment on the two different tight-binding approximations used to calculate the electronic structure and we provide some theory background.

\section{A. Slater Koster approximation}

In most of the calculations in this work we use a multiorbital approach, taking into account the four valence orbitals of the carbon atom, $s, p_{x}, p_{y}$, and $p_{z}$, similar to what was used in previous work. ${ }^{31,42-45}$ Thus, counting the spin, the single particle basis has 8 elements per carbon atom. In addition, we passivate the edge carbon atoms with a single hydrogen atom for which a single $s$ orbital, with the corresponding spin degeneracy, is included. The matrix elements of the Hamiltonian are computed according to the Slater Koster approach considering only first neighbor hoppings. For simplicity we approximate the overlap matrix as the unit matrix. We model both the carbon-carbon and carbon-hydrogen hoppings of graphene with a set of tight-binding parameters derived by Kaschner et $a l .{ }^{46}$ from comparison with density functional calculations. We show these parameters in Table I.

Spin-orbit coupling is treated as an intra-atomic potential:

$$
\mathcal{V}_{\mathrm{SO}}=\lambda \vec{S} \cdot \sum_{I} \vec{L}_{I},
$$

where $\lambda$ is the spin-orbit coupling parameter, $\vec{S}$ is the spin operator, and $\vec{L}_{i}$ is the orbital angular momentum operator acting on the atomic orbitals of site $I$. The representation of this operator in the basis $p_{x}, p_{y}$, and $p_{z}$ is provided in the Appendix. Whereas there is no consensus regarding the value of the atomic spin-orbit coupling in carbon, the values reported in recent work range between $\lambda=4$ and $\lambda=8 \mathrm{meV}^{31,32,36} \mathrm{In}$ this work we always discuss our results for values of $\lambda$ in that range and, when some physical insight is gained by so doing, for values of $\lambda$ much above the realistic range.

\section{B. One-orbital tight-binding model}

The low energy physics of most graphene based nanostructures can be described with a tight-binding model with a single orbital per atom, which can be taken as a $l=1$ atomic orbital projected along the local normal direction to the graphene surface, the so-called $\pi$ orbitals. From the discussion above, it is apparent that the atomic spin-orbit operator mixes orbital states in the same atom with different values of $m$. However, in some instances it is still possible to describe the low energy sector of graphene with an effective Hamiltonian governed by the $\pi$ orbitals in which spin-orbit gives rise to spin dependent hopping terms. ${ }^{11,12}$ We express the effective Hamiltonian using

TABLE I. Slater-Koster parameters and on-site energies involving the same atoms, carbon-carbon interaction, and two different atoms, carbon-hydrogen interaction. All the values are in $\mathrm{eV}$.

\begin{tabular}{lcccr}
\hline \hline & $V s s \sigma$ & $V s p \sigma$ & $V p p \sigma$ & $V p p \pi$ \\
\hline C-C & -7.76 & 8.16 & 7.48 & -3.59 \\
C-H & -6.84 & 7.81 & & \\
& $\varepsilon_{s}^{\mathrm{C}}=-8.8$ & $\varepsilon_{p}^{\mathrm{C}}=0.0$ & $\varepsilon_{s}^{\mathrm{H}}=-2.5$ & \\
\hline \hline
\end{tabular}

second quantization operators $c_{I, \sigma}^{\dagger}$ that create one electron in the atomic site $I$ with spin $\sigma$ :

$$
\mathcal{H}_{0}=\sum_{I, J, \sigma, \sigma^{\prime}} \mathcal{T}_{I \sigma, J \sigma^{\prime}} c_{I, \sigma}^{\dagger} c_{J, \sigma^{\prime}}
$$

The Hamiltonian matrix is the sum of four terms:

$$
\begin{aligned}
\mathcal{T}_{I \sigma, J \sigma^{\prime}}= & t \delta_{\sigma, \sigma^{\prime}} N_{I, J}^{(1)}+t^{\prime} \delta_{\sigma, \sigma^{\prime}} N_{I, J}^{(2)} \\
& +i t_{\mathrm{KM}} \vec{\tau}_{\sigma, \sigma^{\prime}} \cdot\left(\vec{d}_{1} \times \vec{d}_{2}\right) N_{I, J}^{(2)}
\end{aligned}
$$

The elements of the matrix $N_{I, J}^{(1)}\left(N_{I, J}^{(2)}\right)$ are equal to 1 when $I$ and $J$ are first (second) neighbors, and zero everywhere else. Thus, the first two terms are the spin independent first and second neighbor hoppings. The third term is the Kane-Mele spin-orbit model. ${ }^{11,12}$ It is a spin dependent second neighbor hopping between sites $I$ and $J$ which have a common first neighbor $C$. The unit vector along the bond between sites $I$ and $C(C$ and $J)$ is denoted by $\vec{d}_{1}\left(\vec{d}_{2}\right)$. In the Kane-Mele spin-orbit model the spin dynamics is linked to the bond orientation. Thus, in flat graphene and graphene ribbons, the bonds lie in a plane so that the Kane-Mele spin-orbit conserves the spin along the normal to the plane. This is in contrast to the curved ribbons and nanotubes considered below, for which the bond vectors are not restricted to a plane and no component of the spin operator is conserved. In the rest of this paper we calculate the band structure of graphene based one-dimensional structures using the four-orbital Slater Koster model and compare with the results of the one-orbital model defined by Eqs. (2) and (3). Whereas the one-orbital model gives results very similar to those of the flat ribbon, this is not so in the case of curved ribbons.

\section{Some general results}

We study one-dimensional ribbons formed repeating a crystal unit cell. Taking advantage of the crystal symmetry, the Hamiltonian can be written as $\sum \mathcal{H}_{n, m}(\vec{k})$ where the indexes $n$ and $m$ run over the single-particle spin orbitals of the unit cell. For the one-dimensional structures considered below, the unit cell is shown in Fig. 1. The period of the crystal is given by the graphene lattice parameter $a, \vec{k}$ is given by a scalar $k$, and the Brillouin zone can be chosen in the interval $-\pi / a$ and $\pi / a$. The eigenstates of the crystal are labeled with the band index

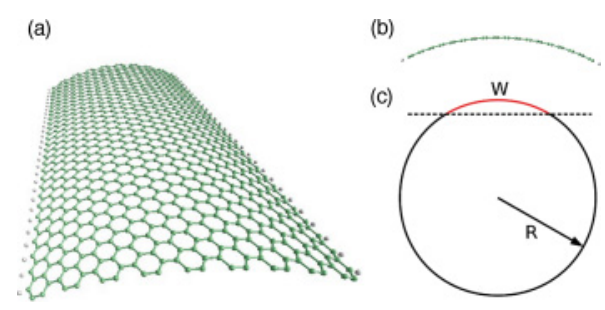

FIG. 1. (Color online) (a) Perspective view of a curved graphene ribbon with edge atoms passivated with hydrogen. (b) Section of a curved ribbon. (c) Scheme of the procedure to generate curved ribbons. 
$v$ and their crystal momentum $k$. They are linear combination of the atom $I$ with quantum numbers $n=l, m$ :

$$
\Psi_{\nu k}(N, \vec{r})=e^{i k N a} \sum_{I, n, s} C_{\nu k}(I, n, s) \phi_{n}\left(\vec{r}-\vec{r}_{I}\right) \chi_{s},
$$

where $N$ is an integer that labels the unit cell, and $\phi_{n}\left(\vec{r}-\vec{r}_{I}\right)$ is the atomic orbital with orbital quantum numbers $n$ (which encodes $l$ and $m$ ) in the atom $I$ of the unit cell. The eigenstate of the spin operator along the $z$ axis is denoted by $\chi_{s}$, where $s$ can take values $\pm \frac{1}{2}$. Because of the spin-orbit coupling it is important to specify the positions of the atoms with respect to the spin quantization axis.

Due to time reversal symmetry, every state with energy $\epsilon_{v}(k)$ must have the same energy as its time reversal partner, $\epsilon_{v^{\prime}}(-k)$, where $v$ and $v^{\prime}$ label states related by time reversal symmetry. In systems with inversion symmetry the bands satisfy $\epsilon_{v}(k)=\epsilon_{v^{\prime}}(k)$ so that, in the same $k$ point, there are at least two degenerate states. In systems without inversion symmetry, like the curved ribbons considered below, a twofold degeneracy at a given $k$ point is not warranted. In this nondegenerate situation we can compute, without ambiguity, the spin density associated with a given state with quantum numbers $v, k$ as

$$
\left\langle\vec{S}_{v, k}(I)\right\rangle \equiv \sum_{i, n, s, s^{\prime}} C_{k, v}^{*}(I, n, s) C_{k, v}\left(I, n, s^{\prime}\right) \vec{S}_{s, s^{\prime}},
$$

where $\vec{S}_{\sigma, \sigma^{\prime}}$ are the Pauli spin $1 / 2$ matrices.

In the cases with inversion symmetry, like the flat ribbon considered below, for a given $k$ point there are at least two degenerate bands. Thus, any linear combination of states of the degenerate pair $\Psi_{v k}$ and $\Psi_{v^{\prime} k}$ is also the eigenstate of the Hamiltonian and has different spin density. In these instances, we include an infinitesimally small magnetic field in the calculation which breaks the degeneracy and permits us to attribute a given spin density to a given state. When the calculated spin densities so obtained are independent of the orientation of the infinitesimally small magnetic field, they can be considered intrinsic properties of the spin states. As we discuss below, this is the case of the spin filter states in flat spin ribbons, which point perpendicular to the ribbon and have a strong correlation between spin orientation, edge, and velocity, as predicted by Kane and Mele. ${ }^{11,12}$

In order to characterize the properties of a given state it will also be convenient to calculate their sublattice polarization:

$$
\left\langle\sigma_{v, k}^{z}\right\rangle=\sum_{I, n, s}\left|C_{k, v}(I, n, s)\right|^{2} \sigma_{z}(I),
$$

where $\sigma_{z}(I)=+1$ when $I$ is an $A$ site and $\sigma_{z}(I)=-1$ when $I$ is a $B$ site.

\section{FLAT GRAPHENE ZIGZAG RIBBONS}

\section{A. Two-dimensional graphene}

The spin properties and electronic structure of the flat ribbons considered below can be related to those of the twodimensional graphene crystal. We briefly recall the spin-orbit physics of two-dimensional graphene as described within the Slater Koster model. ${ }^{31}$ Within this approach, the electronic structure of two-dimensional graphene is described by a
$16 \times 16$ matrix, corresponding to the two atoms $A$ and $B$ of the unit cell. ${ }^{31}$ At zero spin-orbit the 16 bands are two copies (one per spin) of three bonding-antibonding pairs of $\sigma$ bands and one bonding-antibonding pair of the $\pi$ orbitals which compose the states of the bands at the Fermi energy and are decoupled from the $\sigma$ bands. The Fermi surface is composed of two points $K$ and $K^{\prime}$ where the gap between the two $\pi$ bands vanishes. In the neighborhood of both $K$ and $K^{\prime}$ points the two $\pi$ bands are linear and the $k \cdot p$ theory is formally identical to that of massless two-dimensional Dirac electrons. Spin-orbit couples the $\sigma$ and $\pi$ orbitals, producing anticrossings away from the Fermi energy and opening a gap at the $K$ and $K^{\prime}$ points.

Within the one-orbital approach the Hamiltonian of graphene reads

$$
\mathcal{H}=F(\vec{k}) \sigma_{x} \mathbf{1}+G(\vec{k}) s_{z} \sigma_{z},
$$

where the $\sigma$ operators act on the sublattice space $A$ and $B, \mathbf{1}$ is the unit matrix in the spin space, and $s_{z}= \pm 1$ labels the spin. The first neighbor hopping function reads

$$
F(\vec{k})=t\left(1+e^{i \vec{k} \cdot \vec{a}_{1}}+e^{i \vec{k} \cdot \vec{a}_{2}}\right),
$$

with $\vec{a}_{1}=a(1,0)$ and $\vec{a}_{2}=a\left(\cos \frac{\pi}{3}, \sin \frac{\pi}{3}\right)$. The Kane-Mele second neighbor spin-orbit coupling yields

$$
G(\vec{k})=i t_{\mathrm{KM}}\left(e^{i \vec{k} \cdot \vec{a}_{1}}-e^{i \vec{k} \cdot \vec{a}_{2}}+e^{i \vec{k} \cdot\left(\vec{a}_{2}-\vec{a}_{1}\right)}-\text { h.c. }\right) .
$$

At the Dirac points $K$ and $K^{\prime}$, the function $F(\vec{k})$ vanishes in contrast with the the spin-orbit term, which is given by $G\left(K^{\prime} a\right)=-G(K a)=3 \sqrt{3} t_{\mathrm{KM}}$. Thus, the energy levels of graphene at the Dirac points are governed by the following Hamiltonian: ${ }^{11}$

$$
h=\Delta_{\mathrm{SO}} \tau_{z} \sigma_{z} s_{z},
$$

where $\tau_{z}= \pm 1$ labels the valley quantum number and $\Delta_{\mathrm{SO}}=$ $3 \sqrt{3} t_{\mathrm{KM}}$. Thus, for a given spin orientation $s_{z}= \pm 1$, spinorbit opens a gap that takes a valley dependent value $2\left|\Delta_{\text {SO }}\right|$. This spin and valley dependent gap would make graphene a peculiar type of insulator which could not be connected with a standard insulator by smooth variation of a parameter in the Hamiltonian, ${ }^{11,12}$ i.e., a quantum spin Hall insulator.

\section{B. Flat ribbons}

We now discuss the electronic structure of flat graphene ribbons with zigzag edges. The unit cell that defines the zigzag ribbon has $N$ carbon atoms and two hydrogen atoms that passivate the dangling bonds in the edges of the ribbon. These structures were proposed by Nakada et al. ${ }^{47}$ Using the one-orbital tight-binding model, without spin-orbit coupling, they found that zigzag ribbons have almost flat bands at the Fermi energy, localized at the edges. An important feature of zigzag edges is the fact that all the atoms belong to the same sublattice. Since the honeycomb lattice is bipartite, a semi-infinite graphene plane with a zigzag termination must have zero energy edge states ${ }^{48}$ whose wave function decays exponentially in the bulk, with full sublattice polarization.

In finite width zigzag ribbons, the exponential tails of the states of the two edges hybridize, resulting in a bondingantibonding pair of weakly dispersing bands. ${ }^{17}$ The bands of the zigzag ribbon can be obtained by folding from those of two-dimensional graphene, either with real or imaginary 
transverse wave vector, and the longitudinal wave vector varying along the line that joins the two valleys $K$ and $K^{\prime}$. Thus, the valley number is preserved in zigzag ribbons.

Spin-orbit coupling, described with the one-orbital model, has a dramatic effect on the (four) edge bands. ${ }^{11,12}$ The secondneighbor hopping makes the single edge band dispersive and overcomes the weak interedge hybridization. Interestingly the quantum numbers connect well with those at the Dirac points, which are described by the effective Hamiltonian (9). Thus, as we move from valley $K$ to $K^{\prime}$ (positive velocity bands) the spin $\uparrow(\downarrow)$ states of the edge with sublattice $A(B)$ must change from the top of the valence band to the bottom of the conduction band. The roles of spin and sublattice are reversed when considering the two bands that start at $K^{\prime}$ and end at $K$. Thus spin $\uparrow$ electrons move with positive velocity in one edge and negative velocity in the other.

This scenario is confirmed by the four-orbital model and is expected based on the fact that $2 \mathrm{D}$ graphene with spin-orbit coupling is a quantum spin Hall insulator. In Fig. 2 we show the bands of a flat ribbon with $N=20$ carbon atoms. In Fig. 2(a) we show the bands calculated without spin-orbit coupling. The calculation shows both the edge and confined $\pi$ bands as well as some $\sigma$ bands higher in energy. The rather flat band at $+7 \mathrm{eV}$ comes from the hydrogen $s$ orbitals hybridized with the carbon edge $\sigma$ orbitals. In the inset we zoom on the edge states to show that they are almost dispersionless except when $k$ gets close to the Dirac point. In Fig. 2(b) we show the same edge states, calculated with SO coupling, both within the four-orbital and the one-orbital model. For this particular case we have taken $\lambda=500 \mathrm{meV}$ and $t_{\mathrm{KM}}=0.42 \mathrm{meV}$. Figure 2(c) shows a slightly different slope for valence and conduction bands for the four-orbital case. This electron-hole symmetry breaking can not be captured with the one-orbital Kane-Mele model.

It is apparent that the edge states acquire a linear dispersion $\epsilon=m k$. The slope $m$ of the edge states dispersion increases linearly with the size of the gap at the Dirac points, which in turn scales quadratically with $\lambda$ (and linearly with $t_{\mathrm{KM}}$ ). We
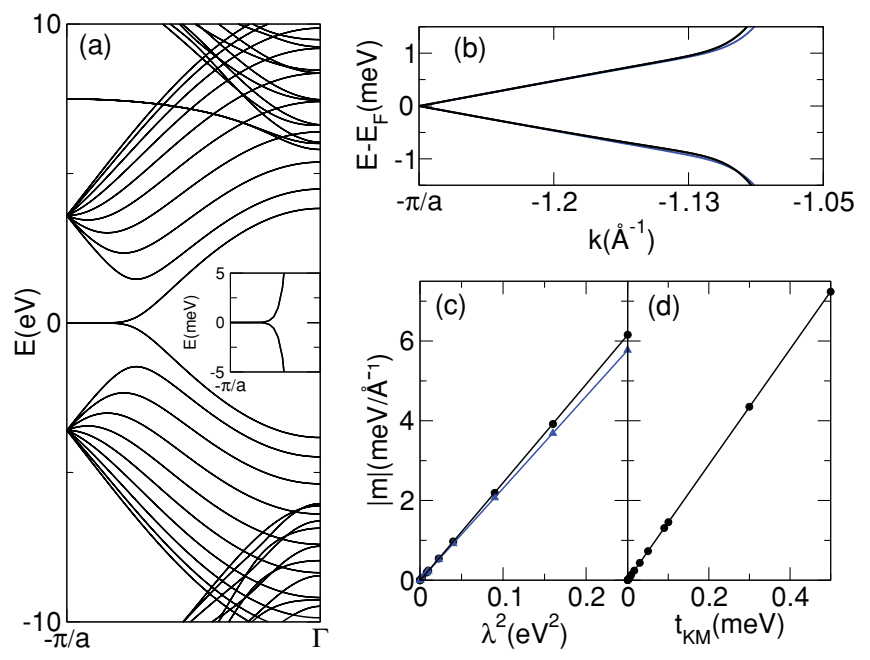

FIG. 2. (Color online) (a) Bands for flat ribbon without SO. Inset: Zoom of the edge bands for case (a). (b) Edge bands with SO $(\lambda=$ $500 \mathrm{meV}$ ) and $t_{\mathrm{KM}}=0.42 \mathrm{meV}$. (c) Slope vs $\lambda^{2}$. (d) Slope vs $t_{\mathrm{KM}}$.
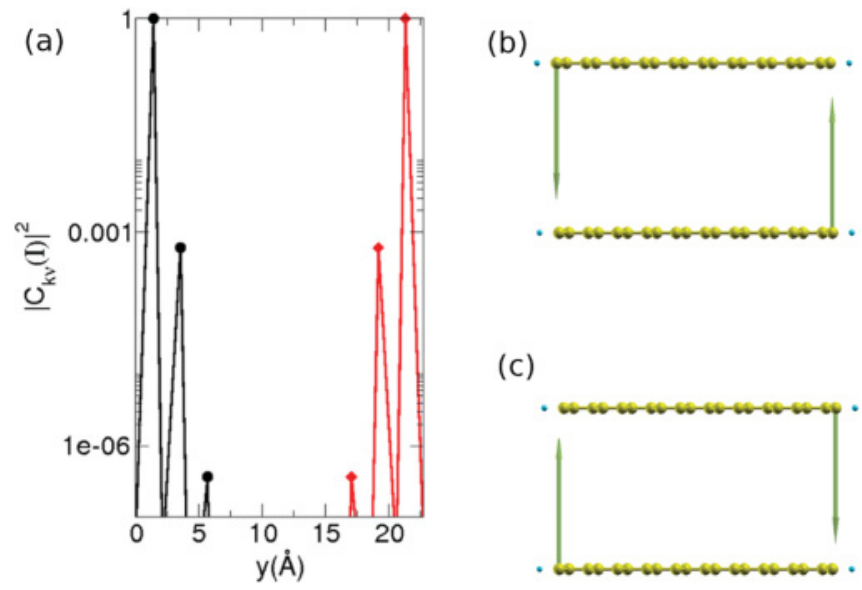

FIG. 3. Properties of the edge state valence band. (a) Square of the Wave function for valence states with $k=\frac{\pi}{a}-0.01$. The sublattice polarization is apparent. (b) Spin density for states with $k=\frac{\pi}{a}-$ 0.01 . (c) Spin density of time reversal symmetric state with $k=-\frac{a}{a}+$ 0.01 . The spin density of the four states is polarized perpendicular to the sample.

can use $m$ to quantify the effect of spin-orbit coupling on the edge states. Figure 2(d) shows that for flat ribbons, we can fit $m=\alpha \lambda^{2}$ with $\alpha \simeq 24 \frac{\AA}{\mathrm{eV}^{2}}$.

Since the flat ribbons have inversion symmetry, the bands have a twofold degeneracy. In order to avoid numerical spin mixing of the degenerate states we apply a tiny magnetic field (always less than $2 \times 10^{-4} \mathrm{~T}$ ) to split the states. As long as the associated Zeeman splitting is negligible compared to the spin-orbit coupling, the direction of the field is irrelevant. By so doing, we can plot the spin density of the edge states without ambiguity. In Fig. 3 we show both the spin density of the four edge states with $k=\frac{\pi}{a}-0.01$ and the square of the wave function for valence states, calculated with the fourorbital model. In agreement with the one-orbital Kane-Mele model, the spin densities are peaked in the edge, oriented perpendicular to the plane of the ribbon. We have repeated the calculation rotating the plane of the ribbon and obtained the same result. From inspection of Figs. 2(b) and 3, it is apparent that the valence bands correspond to the edge states and display the spin filter effect; i.e., in a given edge right goers and left goers have opposite spin.

\section{CURVED GRAPHENE ZIGZAG RIBBONS}

The calculation of the previous section, using the fourorbital model, backs up the conclusions of the Kane-Mele oneorbital model for the spin filter effect in graphene. However, the bandwidth of the edge states is less than $0.1 \mu \mathrm{eV}$ for the accepted values ${ }^{43}$ of $\lambda=5 \mathrm{meV}$. Thus, the effect is very hard to observe in flat graphene ribbons. This leads us to consider ways to enhance the effect of spin-orbit. For that matter, we calculate the edge states in a curved graphene zigzag ribbon, similar to those reported recently, ${ }^{39,40,49}$ using the four-orbital model. In contrast to flat ribbons, the properties of the edge states of a curved ribbon cannot be inferred from those of a parent two-dimensional compound, because it is not possible 

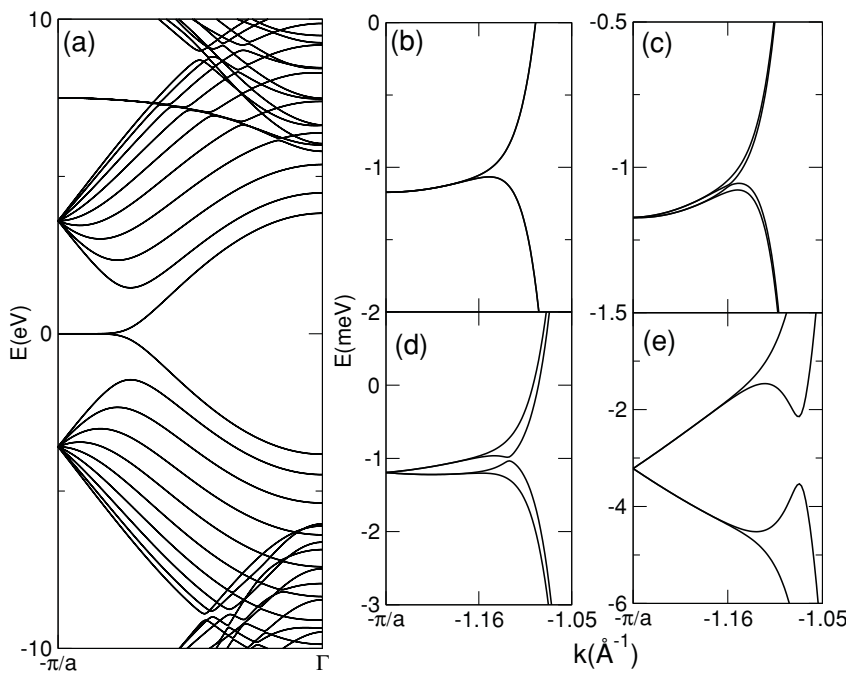

FIG. 4. Electronic structure of curved zigzag ribbon with $R=$ $4.1 \mathrm{~nm}$. (a) Bands for curved ribbon without SO. (b)-(e) Zoom of the edge bands for $\lambda=0$ (b), $\lambda=5 \mathrm{meV}$ (c), $\lambda=50 \mathrm{meV}$ (d), and $\lambda=500 \mathrm{meV}(\mathrm{e})$.

to define a two-dimensional crystal with a finite unit cell and constant curvature.

The unit cell of the curved ribbons is obtained as fraction of a $(n, n)$ nanotube, with radius $R$ (see Fig. 1$)$. For a given nanotube we can obtain a series of curved ribbons with the same curvature $R^{-1}$ and different widths $W$ or different numbers of carbon atoms $N$. We can also study ribbons with the same $N$ and different curvatures $R$ using a parent nanotube with different $n$. Our curved ribbons are thus defined by $W$ and $R$ or, more precisely, by $n$ and $N$.

\section{A. Energy bands}

The energy bands of curved ribbons is shown in Fig. 4 for a ribbon with $N=20$ and $R=4.1 \mathrm{~nm}$. There are three main differences with the flat ribbon. First, the edge states are dispersive even with $\lambda_{\text {SO }}=0$, as seen in Fig. 4(b). This effect can be reproduced, within the one-orbital model, including an effective second-neighbor hopping $\left(t^{\prime} \simeq-1.5 \mathrm{meV}\right)$. This dispersion breaks electron-hole symmetry and competes with the one induced by SO coupling, as seen in panels (c), (d), and (e) of Fig. 4, with $\lambda_{\mathrm{sO}}=5,50$, and $500 \mathrm{meV}$, respectively.

Second, curvature enhances the effect of spin-orbit coupling, as expected. In order to separate the effect of spin-orbit from the effect induced by curvature, we define the differential bands as the energy bands of the curved ribbon at finite $\lambda_{\text {SO }}$, subtracting the bands without spin-orbit:

$$
\tilde{\epsilon}_{v}(k) \equiv \epsilon_{v}(k)-\epsilon_{v}\left(k, \lambda_{\text {SO }}=0\right) .
$$

In Fig. 5(a) we plot the differential bands for the ribbon with $N=20$ and $R=4.1 \mathrm{~nm}$. They are two doubly degenerate linear bands with opposite velocities in the Brillouin zone boundary. Thus, when the effect of curvature alone is subtracted, the dispersion edge states look pretty much like those of the flat ribbons in the region close to the Brillouin zone boundary. Thus, we can also characterize them by the slope of the linear bands, $m$. In Fig. 5(b) we plot that the slope of
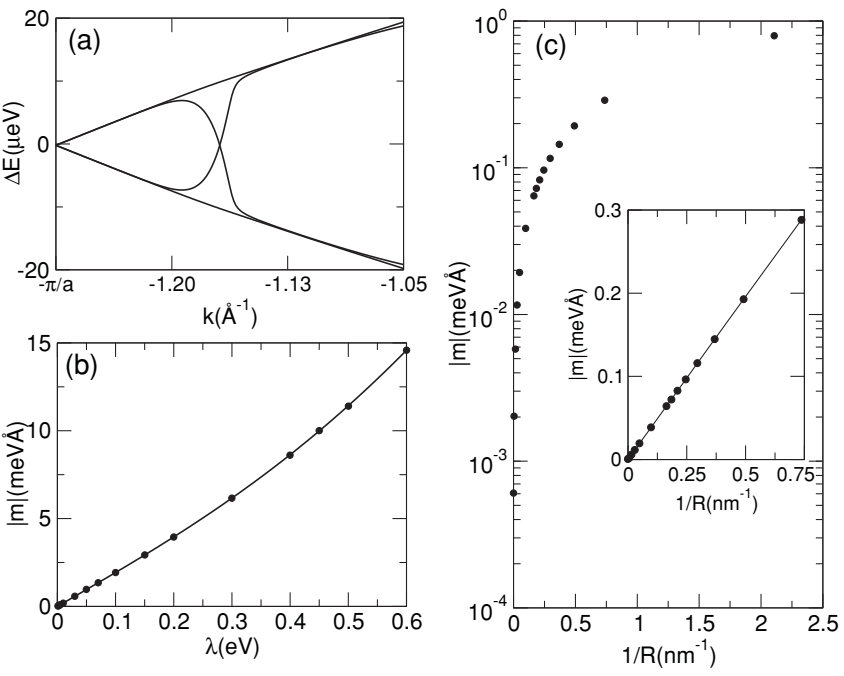

FIG. 5. (a) Differential energy bands [see Eq. (10)] for ribbons with $N=20, R=4.1 \mathrm{~nm}$, and $\lambda=5 \mathrm{meV}$. It is apparent that for small $k$ they are linear, with slope $m$. (b) Slope of the differential energy bands as a function of $\lambda$, for $R=4.1 \mathrm{~nm}$. (c) Slope of the differential energy bands as a function of $\kappa=R^{-1}$, both in linear and logarithmic scale, for $\lambda=5 \mathrm{meV}$. A 100 -fold enhancement of the slope occurs in a very narrow range of small curvatures.

the edge bands as a function of $\lambda$, obtained with the procedure just described, for the same ribbon discussed before $(N=20$, $R=4.1 \mathrm{~nm})$. It is apparent that the slope $m$ is no longer linear in $\lambda^{2}$, in contrast to the case of flat ribbons. Even more interesting, in Fig. 5(c) we plot the slope $m$ for a fixed value of $\lambda=5 \mathrm{meV}$, as a function of the curvature $\kappa=R^{-1}$. We find a dramatic 100 -fold increase at small $\kappa$. This result is consistent with the effective $k \cdot p$ Hamiltonian for carbon nanotubes. ${ }^{7,50}$

The curvature induced enhancement of the spin-orbit effect on the edge states of the curved ribbon can be understood as follows. In flat graphene the Dirac bands are linear combinations of atomic $\pi$ orbitals with quantum numbers $l=$ $1, m=0$. The effect of atomic spin-orbit coupling $\lambda \vec{L} \cdot \vec{S}$ can be understood perturbatively. To first order, spin-orbit coupling has no effect on the product states $|\sigma\rangle \times|l=1, m=0\rangle$. Second order coupling, via intermediate states with $\Delta$ with respect to the Dirac point and orbital quantum numbers $l=1, m= \pm 1$, results in an effective spin-orbit Hamiltonian acting on the $\pi$ orbitals, with strength $\frac{\lambda^{2}}{\Delta}$, which conserves $S_{z}$. Curvature changes this situation, because it mixes the $\pi$ orbitals with the $l=1, m \neq 0$ orbitals, resulting in a spinorbit Hamiltonian for the electrons at the Fermi energy 7,50 linear in the spin-orbit coupling $\lambda$.

The third difference with the flat ribbon is apparent for the bands away from the zone boundary: They are not degenerate. This is shown in Fig. 6(a), which is a zoom of Fig. 4(b), including states at both sides of the Brillouin zone boundary. The lack of degeneracy is originated by the lack of the inversion symmetry of the curved ribbon. Interestingly, the degree of sublattice polarization $\left\langle\sigma_{z}\right\rangle$, shown in Fig. 6(b), anticorrelates with the splitting. In other words, the states strongly localized at the edges are insensitive to the lack of the inversion of the structure, which is nonlocal property. 


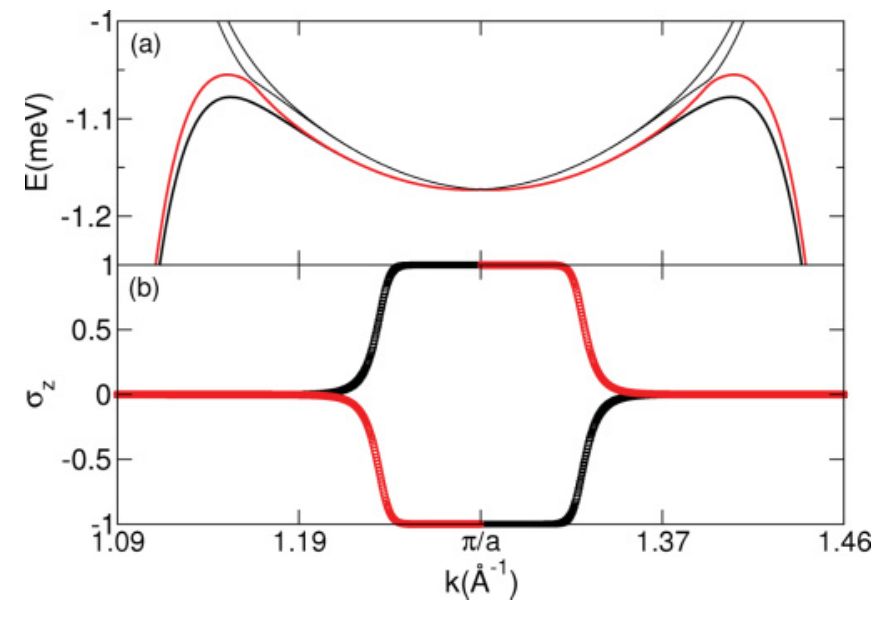

FIG. 6. (Color online) Detail of the edge states for curved ribbon with $R=4.1 \mathrm{~nm}$ and $\lambda=5 \mathrm{meV}$. (a) Dispersion of the edge states. (b) Sublattice polarization $\sigma_{z}$ as a function of $k$ for the two lowest energy bands.

\section{B. Electronic properties}

We now discuss the spin properties of the edge states in the curved ribbons. Note that due to the lack of inversion symmetry, there is no degeneracy at a given $k$ so that the spin density is an intrinsic property of the state. In Fig. 7 we plot the magnetization density $\left\langle\vec{S}_{v, k}(I)\right\rangle$ for the two lowest energy edge bands with momentum $k=\frac{\pi}{a}+0.01$ (upper panels) and $k=$ $-\frac{\pi}{a}-0.01$ (lower panel), for a ribbon with $R=4.1 \mathrm{~nm}$ and $\lambda \stackrel{a}{=} 5 \mathrm{meV}$. Whereas the correlation between the velocity, the spin orientation, and the edge is the same as in flat ribbons, it is apparent that the quantization axis is no longer parallel to the local normal direction. The spin of the edge states lies almost perpendicular to the normal direction. Thus, this is different from the case of nanotubes, where the spin quantization axis is parallel to the tube main axis, and different from the flat ribbon.
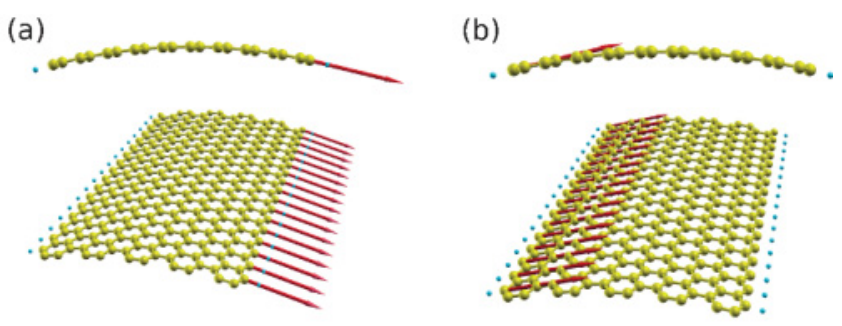

(c)
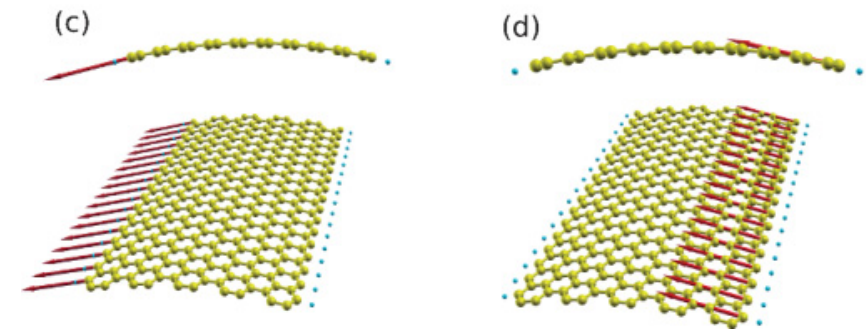

FIG. 7. (Color online) Spin densities for states with $k=-\frac{\pi}{a}+$ 0.01 [panels (a) and (b)] and $k=\frac{\pi}{a}-0.01$ [panels (c) and (d)].
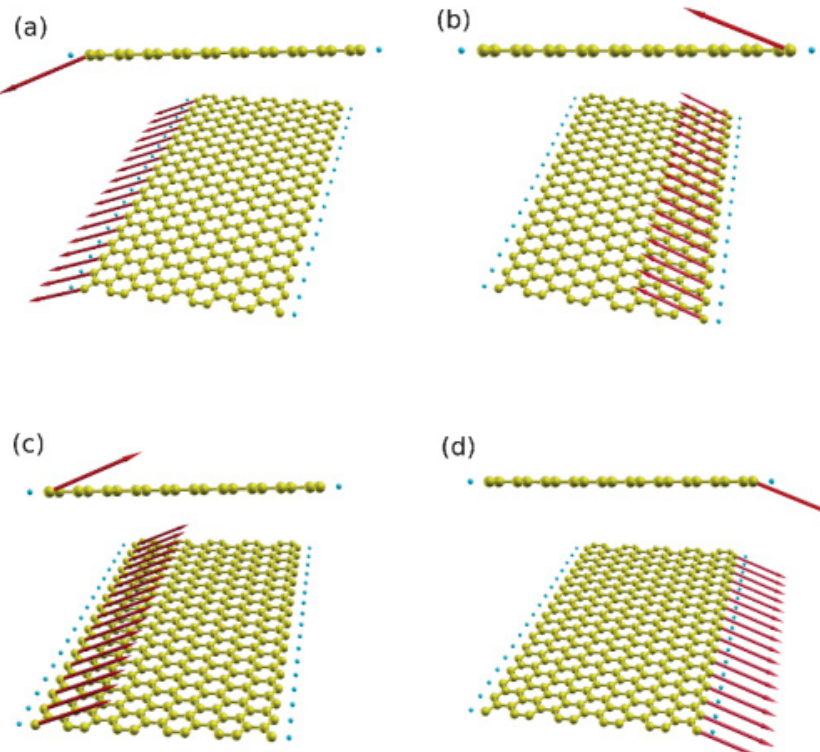

(d)

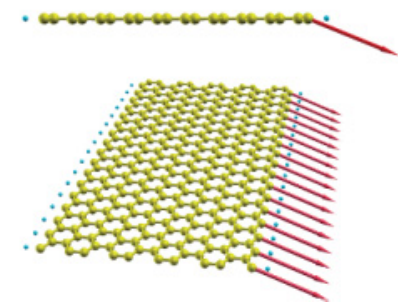

FIG. 8. (Color online) Spin densities for states with $k=-\frac{\pi}{a}+$ 0.01 [panels (a) and (b)] and $k=\frac{\pi}{a}-0.01$ [panels (c) and (d)] in almost flat ribbon, $R^{-1}=3.7 \times 10^{-3} \mathrm{~nm}^{-1}$. Note that curvature cannot be appreciated.

The effect is even more striking in the case of an almost flat ribbon, shown in Fig. 8 for which the spin quantization direction is clearly not perpendicular to the ribbon plane. Thus, a very small curvature is enough to change the spin quantization direction of the edge states. This is better seen in Fig. 9, where we plot the angle formed between the spin quantization axis and the local normal in the edge atom. In Fig. 9(a) we show the evolution of the angle for the states with $k=-\frac{\pi}{a}+0.01$ for the two lowest energy edge bands. For flat ribbons $R^{-1}=0$, the angles are $0^{\circ}$ and $180^{\circ}$, i.e., the quantization axis is perpendicular to the ribbon plane. In the opposite limit, for large curvature, the spin quantization angle lies perpendicular to the normal, i.e., tangential, but

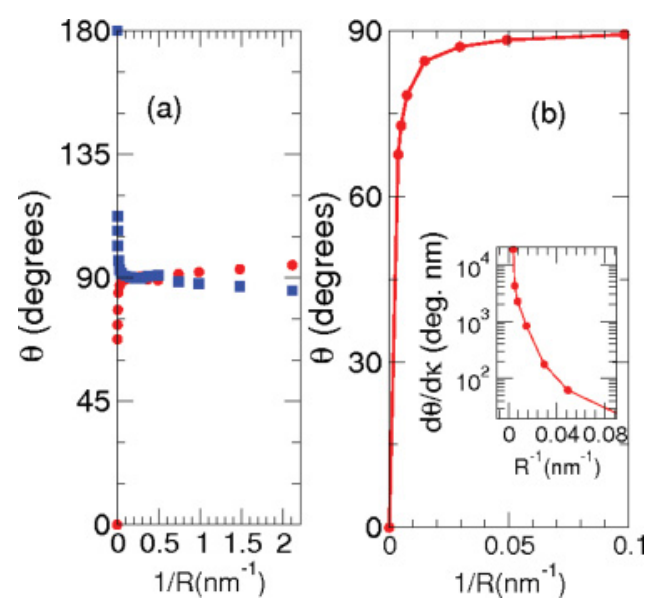

FIG. 9. (Color online) (a) Angle $\theta$ formed by the edge spin density and the local normal as a function of curvature $\kappa=R^{-1}$ for $\lambda=5$ meV. (b) Detail of (a) for small $\kappa$. Inset: derivative of the angle with the curvature. Note the extremely large $\frac{d \theta}{d \kappa}$ for a very narrow region of small curvatures. 
always in the plane perpendicular to the ribbon transport direction.

The transition between the two limits is far from smooth. Even for the less curved ribbon that we have considered, with $R=271 \mathrm{~nm}$, we have $\theta \simeq 70^{\circ}$. This is better seen in Fig. 9(b), where we show the low curvature region only for one of the bands. The dramatic effect of curvature on the spin orientation of the edge states is quantified in the inset of Fig. 9(b), where we show $\frac{d \theta}{d \kappa}$ as a function of curvature $\kappa$. For small curvatures the derivative blows up exponentially. Thus, the spin orientation of the edge states is very sensitive to moderate buckling deformation of the ribbon. This sensitivity is especially important for the small values of $\lambda$ adequate for carbon. Larger values of $\lambda$ reduce the effect. We have verified that the single-orbital model with the generalized Kane-Mele Hamiltonian is not sufficient to capture the effect.

\section{DISCUSSION AND CONCLUSIONS}

We are now in position to discuss whether or not the spin filter effect could be more easily observed in curved graphene ribbons. One side, the bandwidth of the edge states is dramatically increased. For moderate curvatures $(R=4.1 \mathrm{~nm})$ the bandwidth of the edge states is $20 \mu \mathrm{eV}$, and it can reach $60 \mu \mathrm{eV}$ for $R \simeq 1 \mathrm{~nm}$, to be compared with $0.1 \mu \mathrm{eV}$ for flat ribbons. Thus, experiments done at $100 \mathrm{mK}$ could resolve the edge band of curved ribbons. On the other hand, the curvatureinduced second-neighbor hopping breaks the electron-hole symmetry [see Fig. 6(a)], so that now there are four edge bands at the Fermi energy. At each edge there would be left goers and right goers with the same spin orientation, although slightly different $k$. Taken at face value, this would imply that there is no spin current in the ground state and backscattering would not be protected.

In conclusion, we have studied the interplay of spin-orbit coupling and curvature in the edge states of graphene zigzag ribbons. Our main conclusions are as follows:

(1) In the case of the flat graphene ribbon, the microscopic four-orbital model yields results identical to those of the KaneMele one-orbital model. In particular, the edge states have the spin filter property ${ }^{11,12}$ and the spin is quantized perpendicular to the sample.

(2) Curved graphene ribbons also have spin-filtered edge states. The bandwidth of the edge bands of curved ribbons is increased by as much as 100 for moderate curvatures and is proportional to the curvature, for fixed spin-orbit coupling.

(3) Curvature induces a second neighbor hopping which modifies the dispersion of the edge states and, in this sense, competes with their spin-orbit induced dispersion.

(4) The spin of the edge states is not quantized along the direction normal to the ribbon. For moderate curvature, their quantization direction depends very strongly on the curvature of the ribbon. Above a certain curvature, the quantization direction is independent of curvature and perpendicular to both the normal and the ribbon direction. The strong sensitivity of the spin orientation of edge states on the curvature suggests that flexural phonons can be a very efficient mechanism for spin relaxation in graphene.

Note added: During the final stages of this work a related preprint was posted. ${ }^{52}$

\section{ACKNOWLEDGMENTS}

We acknowledge fruitful discussions with David Soriano, A. S. Núñez, and S. Fratini. This work has been financially supported by MEC-Spain (MAT07-67845, FIS2010-21883C02-01, and CONSOLIDER CSD2007-00010) and Generalitat Valenciana (ACOMP/2010/070).

\section{APPENDIX: ATOMIC ORBITAL BASIS AND MATRIX ELEMENTS}

In this appendix we give the expressions for the atomic orbitals and the corresponding angular momentum matrix elements, necessary to compute the spin-orbit matrix. In our formalism, the atomic orbitals are described in terms of the Cartesian basis, $s, p_{x}, p_{y}, p_{z}$, which is related to the basis of the eigenstates of $L^{2}$ and $L_{z}$, through the following transformation: ${ }^{51}$

$$
\begin{gathered}
|s\rangle=|l=0, m=0\rangle, \\
\left|p_{x}\right\rangle=-\frac{1}{\sqrt{2}}(|l=1, m=1\rangle-|l=1, m=-1\rangle, \\
\left|p_{y}\right\rangle=\frac{i}{\sqrt{2}}(|l=1, m=1\rangle+|l=1, m=-1\rangle, \\
\left|p_{z}\right\rangle=|l=1, m=0\rangle .
\end{gathered}
$$

The spin-orbit Hamiltonian operator reads,

$$
\mathcal{V}_{\mathrm{SO}}=\lambda\left[\frac{\hat{L}_{+} \hat{s}_{-}+\hat{L}_{-} \hat{s}_{+}}{2}+\hat{L}_{z} \hat{s}_{z}\right],
$$

which only affects the $l=1$ subspace. The matrix elements of this operator in the Cartesian basis read,

$$
\begin{aligned}
& \left|p_{x}, \uparrow\right\rangle\left|p_{y}, \uparrow\right\rangle\left|p_{z}, \uparrow\right\rangle\left|p_{x}, \downarrow\right\rangle\left|p_{y}, \downarrow\right\rangle\left|p_{z}, \downarrow\right\rangle \\
& \left|p_{x}, \uparrow\right\rangle \quad 0 \quad-i \lambda / 2 \quad 0 \quad 00000 / 2 \\
& \left|p_{y}, \uparrow\right\rangle \quad i \lambda / 2 \quad 0 \quad 0 \quad 0 \quad 0 \quad 0 \quad-i \lambda / 2 \\
& \left|p_{z}, \uparrow\right\rangle \quad 0 \quad 0 \quad 0 \quad-\lambda / 2 \quad i \lambda / 2 \quad 0 \\
& \left|p_{x}, \downarrow\right\rangle \quad 0 \quad 0 \quad-\lambda / 2 \quad 0 \quad i \lambda / 2 \quad 0 \\
& \left|p_{y}, \downarrow\right\rangle \quad 0 \quad 0 \quad-i \lambda / 2-i \lambda / 2 \quad 0 \quad 0 \\
& \left|p_{z}, \downarrow\right\rangle \quad \lambda / 2 \quad-i \lambda / 2 \quad 0 \quad 0000
\end{aligned}
$$

${ }^{1}$ R. Saito, G. Dresselhaus, and M. S. Dresselhaus, Physical Properties of Carbon Nanotubes (Imperial College Press, London, 1998).

${ }^{2}$ C. L. Kane and E. J. Mele, Phys. Rev. Lett. 78, 1932 (1997).
${ }^{3}$ J. C. Meyer et al., Nature (London) 446, 60 (2007).

${ }^{4}$ A. F. Morpurgo and F. Guinea, Phys. Rev. Lett. 97, 196804 (2006). 
${ }^{5}$ S. V. Morozov, K. S. Novoselov, M. I. Katsnelson, F. Schedin, L. A. Ponomarenko, D. Jiang, and A. K. Geim, Phys. Rev. Lett. 97, 016801 (2006).

${ }^{6}$ L. Brey and J. J. Palacios, Phys. Rev. B 77, 041403 (2008).

${ }^{7}$ D. Huertas-Hernando, F. Guinea, and Arne Brataas, Phys. Rev. B 74, 155426 (2006).

${ }^{8}$ Z. L. Wang and J. Song, Science 312, 242 (2006).

${ }^{9}$ P. Fei, P. H. Yeh, J. Zhou, S. Xu, Y. Gao, J. Song, Y. Gu, Y. Huang, and Z. Lin Wang, Nano Lett. 9, 3435 (2009).

${ }^{10}$ J. I. Sohn, H. J. Joo, D. Ahn, H. H. Lee, A. E. Porter, K. K. Kim, D. J. Kang, and M. E. Welland, Nano Lett. 9, 3392 (2009).

${ }^{11}$ C. L. Kane and E. J. Mele, Phys. Rev. Lett. 95, 226801 (2005).

${ }^{12}$ C. L. Kane and E. J. Mele, Phys. Rev. Lett. 95, 146802 (2005).

${ }^{13}$ M. Fujita, K. Wakabayashi, K. Nakada, and K. Kusakabe, J. Phys. Soc. Jpn. 65, 1920 (1996).

${ }^{14}$ Y.-W. Son, M. L. Cohen, and S. G. Louie, Phys. Rev. Lett. 97, 216803 (2006).

${ }^{15}$ Y.-W. Son, M. L. Cohen, and S. G. Louie, Nature (London) 444, 347 (2006).

${ }^{16}$ D. Gunlycke et al., Nano Lett. 7, 3608 (2007).

${ }^{17}$ J. Fernández-Rossier, Phys. Rev. B. 77, 075430 (2008).

${ }^{18}$ O. V. Yazyev and M. I. Katsnelson, Phys. Rev. Lett. 100, 047209 (2008).

${ }^{19}$ W. Y. Kim and K. S. Kim, Nature Nanotechnol. 3, 408 (2008).

${ }^{20}$ F. Muñoz-Rojas, J. Fernández-Rossier, and J. J. Palacios, Phys. Rev. Lett. 102, 136810 (2009).

${ }^{21}$ J. Jung, T. Pereg-Barnea, and A. H. MacDonald, Phys. Rev. Lett. 102, 227205 (2009).

${ }^{22}$ D. Soriano and J. Fernández-Rossier, Phys. Rev. B 82, 161302(R) (2010).

${ }^{23}$ X. Li, X. Wang, L. Zhang, S. Lee, and H. Dai, Science 319, 1229 (2008).

${ }^{24}$ J. Cai, P. Ruffieux, R. Jaafar, M. Bieri, T. Braun, S. Blankenburg, M. Muoth, A. P. Seitsonen, M. Saleh, X. Feng, K. Mullen, and R. Fasel, Nature (London) 466, 470 (2010).

${ }^{25}$ C. Tao, L. Jiao, O. V. Yazyev, Y. C. Chen, J. Feng, X. Zhang, R. B. Capaz, J. M. Tour, A. Zettl, S. G. Louie, H. Dai, and M. F. Crommie, e-print arXiv:1101.1141 (to be published).

${ }^{26}$ P. Esquinazi, A. Setzer, R. Hohne, C. Semmelhack, Y. Kopelevich, D. Spemann, T. Butz, B. Kohlstrunk, and M. Losche, Phys. Rev. B 66, 024429 (2002).

${ }^{27}$ P. Esquinazi, D. Spemann, R. Höhne, A. Setzer, K.-H. Han, and T. Butz, Phys. Rev. Lett. 91, 227201 (2003).

${ }^{28}$ Yan Wang, Yi Huang, You Song, Xiaoyan Zhang, Yanfeng Ma, Jiajie Liang, and Yongsheng Chen, Nano Lett. 9, 220 (2009).
${ }^{29}$ J. Cervenka, M. I. Katsnelson, and C. F. J. Flipse, Nature Phys. 5, 840 (2009).

${ }^{30}$ M. A. Ramos, J. Barzola Quiquia, P. Esquinazi, A. Muñoz-Martin, A. Climent-Font, and M. García-Hernández, Phys. Rev. B 81, 214404 (2010).

${ }^{31}$ Hongki Min, J. E. Hill, N. A. Sinitsyn, B. R. Sahu, Leonard Kleinman, and A. H. MacDonald, Phys. Rev. B 74, 165310 (2006).

${ }^{32}$ Y. Yao, F. Ye, X. L. Qi, S. C. Zhang, and Z. Fang, Phys. Rev. B 75, 041401(R) (2007).

${ }^{33}$ M. Gmitra, S. Konschuh, C. Ertler, C. Ambrosch-Draxl, and J. Fabian, Phys. Rev. B 80, 235431 (2009).

${ }^{34}$ N. Tombros, J. Csaba, M. Popinciuc, J. Mihaita, H. T. Jonkman, and B. J. van Wees, Nature (London) 448, 571 (2007).

${ }^{35}$ O. V. Yazyev, Nano Lett. 8, 1011 (2008).

${ }^{36}$ F. Kuemmeth, S. Ilani, D. C. Ralph, and P. L. McEuen, Nature (London) 452, 448 (2008).

${ }^{37}$ D. C. Elias et al., Science 323, 610 (2009).

${ }^{38}$ A. H. Castro Neto and F. Guinea, Phys. Rev. Lett. 103, 026804 (2009).

${ }^{39}$ D. V. Kosynkin, A. L. Higginbotham, Alexander Sinitskii, J. R. Lomeda, A. Dimiev, B. K. Price, and J. M. Tour, Nature (London) 458, 872 (2009).

${ }^{40}$ L. Jiao et al., Nature (London) 458, 877 (2009).

${ }^{41}$ A. G. Cano-Márquez et al., Nano Lett. 9, 1527 (2009).

${ }^{42}$ L. Chico, M. P. López-Sancho, and M. C. Muñoz, Phys. Rev. Lett. 93, 176402 (2004).

${ }^{43}$ W. Izumida, K. Sato, and R. Saito, J. Phys. Soc. Jpn. 78, 074707 (2009).

${ }^{44}$ L. Chico, M. P. López-Sancho, and M. C. Muñoz, Phys. Rev. B 79, 235423 (2009).

${ }^{45}$ Jae-Seung Jeong and Hyun-Woo Lee, Phys. Rev. B 80, 075409 (2009).

${ }^{46}$ D. Porezag, Th. Frauenheim, Th. Köhler, G. Seifert, and R. Kaschner, Phys. Rev. B 51, 12947 (1995).

${ }^{47}$ Kyoko Nakada, Mitsutaka Fujita, Gene Dresselhaus, and Mildred S. Dresselhaus, Phys. Rev. B 54, 17954 (1996).

${ }^{48}$ J. J. Palacios, J. Fernández-Rossier, and L. Brey, Phys. Rev. B 77, 195428 (2008).

${ }^{49}$ H. Santos, L. Chico, and L. Brey, Phys. Rev. Lett. 103, 086801 (2009).

${ }^{50}$ T. Ando, J. Phys. Soc. Jpn. 69, 1757 (2000).

${ }^{51}$ C. Cohen-Tannoudji, B. Diu, and F. Laloe, Quantum Mechanics, Vol. 1 (John Wiley and Sons, New York, 1977).

${ }^{52}$ M. P. López-Sancho and M. C. Muñoz, e-print arXiv:1009.5560 (to be published). 